\title{
Improved Content-Based Watermarking Using Scale-Invariant Feature Points
}

\author{
$\mathrm{Na} \mathrm{Li}{ }^{1,2, \star}$, Edwin Hancock ${ }^{2}$, Xiaoshi Zheng ${ }^{1}$, and Lin $\operatorname{Han}^{2}$ \\ 1 Shandong Computer Science Center, \\ Shandong Provincial Key Laboratory of computer Network, Ji'nan, 250014, China \\ lina@keylab.net \\ http://www.scsc.cn/ \\ 2 Department of Computer Science, University of York, \\ Deramore Lane, York, YO10 5GH, UK
}

\begin{abstract}
For most HVS(Human Visual System) perceptual models, the JND(Just Noticeable Difference) values in highly-textured image regions have little difference with those in edge areas. This is not consistent with the characteristics of human vision. In this paper, an improved method is introduced to give a better content-based perceptual mask than traditional ones using the arrangement of scale-invariant feature points. It could decrease the JND values in edge areas of those traditional masks so that they have an obvious difference with values in highly textured areas. Experimental results show the advantages of this improved approach visually, and the enhancement of the invisibility of watermarks.
\end{abstract}

Keywords: content-based watermarking, scale-invariant feature transform, density-based clustering.

\section{Introduction}

With the increasing use of the Internet and the effortless copying, tampering and distribution of digital data, copyright protection for multimedia data has become an important issue. Digital watermarking has emerged [1 as a tool for protecting multimedia data from copyright infringement. Efficient techniques for image watermarking must accurately balance two contrasting requirements. On the one hand, the hidden watermark should be imperceivable to the HVS, and on the other hand, a watermark should not be inserted into image regions which are not perceptually important [2]. Furthermore the watermark must be robust against intentional and unintentional attacks. Robustness and imperceptibility of watermarks are at odds with one-another. In order to ensure an optimal trade-off, HVS perceptual models have found widespread applications in watermarking.

\footnotetext{
* Shandong Province Natural Science Foundation(No.ZR2009GM025,ZR2010FQ018), Shangdong Province Young and Middle-Aged Scientists Research Awards Fund(No. 2008BS01019).
} 
A variety of HVS models developed from image compression and quality assessment [345], have been applied to the design and optimization of digital watermarking since the late 1990's 677. Generally, most of HVS models create a perceptual mask or a JND mask using a multi-channel visual decomposition suggested by psychophysics experiments. A JND mask indicates the maximum amount one can add or subtract at every pixel position of an image without producing any visible difference. This is a so called masking effect. In addition, most watermarking techniques aim at the optimization of the robustness-invisibility trade-off, and are motivated by qualitative perceptual models rather than quantitative visual models.

\section{Literature Review}

HVS models can be applied in the spatial or frequency domain (DFT, DCT, DWT). In general, watermark embedding can be represented by following equation:

$$
y=x+\alpha w
$$

where $y$ is the watermarked image, $x$ is the original image. The embedding can be applied in either the time-spatial domain, or the coefficients in the frequency domain. Here $\alpha$ controls the embedding strength. The aim of the HVS model and the perceptual mask is to optimize embedding strength $\alpha$ so that the best tradeoff can be obtained between the robustness and invisibility of the watermark.

Delaigle et al. 8] present an additive watermarking technique in the Fourier domain. The core of the embedding process is a masking criterion that guarantees the invisibility of the watermark. This perceptual model is derived from Michelson's contrast $C$, which is defined as:

$$
c=\frac{L_{\max }-L_{\min }}{L_{\max }+L_{\min }}
$$

where $L_{\max }$ and $L_{\min }$ are respectively the maximal and minimal luminance value of grating. The masking criterion is depicted as a general expression of the detection threshold contrast.

Another HVS Fourier domain mask is described in [9]. Here Florent et al. introduce a perceptual model by taking into account advanced features of the HVS identified from psychophysics experiments. This HVS model is used to create a perceptual mask and optimize the watermark strength. This is done by combining the perceptual sub-band decomposition of Fourier spectrum with the quantization noise visibility based on the local band limited contrast. Experimental results demonstrate that this method can resist many attacks, including geometrical distortions.

The Watson model [10] is a popular HVS model for DCT domain. It assesses image visual quality by estimating the final perceptual masking threshold used for image compression. According to the mechanisms of the HVS, three factors are considered in the Watson model in order to comprehensively approximate the 
perceptual quality of an image. The three factors are: a) a frequency sensitivity function, b) a luminance masking function, and c) a contrast masking function. A pooling process is used then to combine all the estimated local perceptual distances together to achieve a global perceptual distance.

Lewis et al. 11] tackle the problem of DWT coefficient quantization for compression and propose to adapt the quantization step of each coefficient according to the local noise sensitivity of the eye. Barni et al. 12 make some modifications of the model proposed in [11] in order to better fit the behavior of the HVS to the watermarking problem. A number of factors are taken into account, including luminance, the frequency band, texture and proximity to an edge. Podilchuk et al. 13 propose an image adaptive watermarking algorithm for both the DCT and DWT domains. The JND masks applied to the DCT domain are computed from quantization matrices established, while the JND masks used in the DWT domain are computed from visual thresholds given by Watson et al. 14. For both embedding domains, the watermark robustness has been tested against JPEG compression and cropping.

Huiyan Qi et al. [15] design a perceptual mask in the spatial domain using image features such as the brightness, edges, and region activities. In their research, the exact mask values of the cover image can be obtained, guaranteeing the maximum-possible imperceptivity of the watermark. Therefore, the watermark embedding directly substitutes the final mask for watermark strength $\alpha$. The authors also successfully extend the proposed spatial masking to the DCT domain by searching the extreme value of a quadratic function subject to the bounds on the variable.

In the above-mentioned papers, the computational complexity for the perceptual masks complicates the analysis of the results. Experimental results support the robustness of these approaches. By contrast Voloshynovskiy et al. [16] propose and verify a general perceptual mask referred to as the Noise Visibility Function (NVF), which is based on the Maximum Aposteriori Probability (MAP) estimation and Markov random fields. It is simple, practical and has been widely used in many watermarking algorithms, both in the spatial and frequency domains.

In this paper, we describe an improved perceptual mask using an arrangement of scale-invariant feature points. The approach can decrease the JND values in edge regions so that they have given obvious difference in highly textured areas. Therefore, the improved mask is more suitable for human visual characteristics, with low JND values in both edge and flat areas, and high values only in highly textured regions. We choose the perceptual mask in [16] as the prototype. However the proposed improvement also applies to alternative perceptual masks where the JND values in highly textured area have an insignificant difference with the JND values in edge regions. The remainder of this paper is organized as follows. Section 3 introduces the perceptual model in [16]. Section 4 introduces Scale-Invariant Feature Transform (SIFT). Section 5 presents our improved mask and estimation method. Section 6 gives experimental results. The paper is concluded in Section 7 . 


\section{Original HVS Model}

The perceptual mask in [16] is based on the computation of NVF that characterizes the local image properties, identifying textured and edge regions. In order to determine the final NVF, the authors consider the watermark as noise and estimate it using a classical MAP image denoising approach. We will not provide the details of this theory here, but rather give the main formula of the method and explain its deficiency.

The authors examine two NVFs, a non-stationary Gaussian model and a stationary generalized Gaussian model, and finally propose a stochastically empirical expression for the optimal NVF. This is widely used in image restoration applications, and calculated using the equation:

$$
N V F(i, j)=\frac{1}{1+\theta \cdot \sigma_{x}^{2}(i, j)}
$$

where $\sigma_{x}^{2}(i, j)$ denotes the grey-scale local variance for neighboring pixels. The parameter $\theta$ is used for tuning and plays the role of contrast adjustment in NVF. This version of NVF is the basic prototype for a large quantity of adaptive regularization algorithms. The parameter $\theta$ depends on the image variance and is given by:

$$
\theta=\frac{D}{\sigma_{\max }^{2}(i, j)}
$$

where $D \in[50,100]$ is an experimentally determined constant, and $\sigma_{\max }^{2}(i, j)$ is the maximum local variance for a given image.

Using NVF, the perceptual mask is:

$$
\Lambda=\alpha \cdot(1-N V F)+\beta \cdot N V F
$$

and the watermark embedding equation is:

$$
y=x+\alpha \cdot(1-N V F) \cdot w+\beta \cdot N V F \cdot w
$$

where $\beta$ can be set to 3 for most of real world and computer generated images. The watermark strength parameter $\alpha$ approaches 1 in highly textured areas and approaches 0 in flat region. The third term in equation (6) is added to increase the watermark strength in very flat regions to a level below the visibility threshold. This avoids the problem that the watermark information is (nearly) lost in these areas. The method is illustrated in Fig 1.

As shown in Fig.1, the watermarking rule (Equ.6) embeds the watermark in highly textured areas and areas containing edges stronger than in very flat regions. The deficiency of the method is the relative extraction of the edge and texture information. The JND values in these two regions are very close and the difference between them is not significant for the original HVS model in [16](as illustrated in Fig.1).

From the literature $[13,15,16]$, we note the following two rules consistent with the characteristics of human vision, which are the foundation of the improved method described in this paper: 


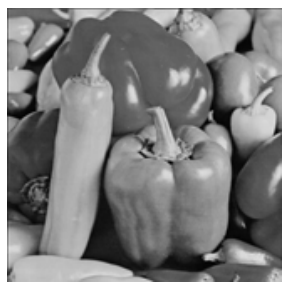

(a)

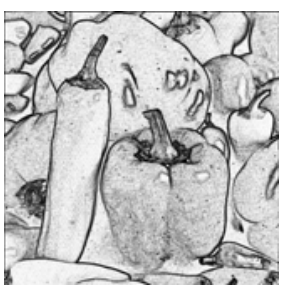

(b)

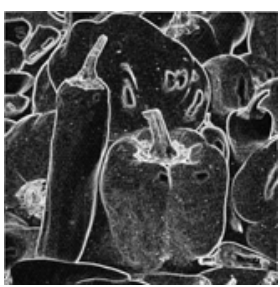

(c)

Fig. 1. Effect of HVS model in [16]. (a) original image(size of $512 \times 512$ ); (b) NVF image ; (c) final perceptual mask, here $\alpha=250$ and $\beta=3$.

- disturbances are less visible in highly textured regions than in flat areas.

- Edges and contours are more sensitive to noise addition than highly textured regions, and less than but close to the very flat areas.

That is to say, the distortion visibility is low in highly textured areas. However it is high in both edge and very flat region. Therefore, only highly textured areas are strongly suited to watermark embedding and the JND values corresponding to these areas must be high. By contrast, edge areas have the low JND values and very flat areas that contain little (or no) frequency information have the lowest JND values. The perceptual mask in [16] follows just the first of two rules. It is therefore not consistent with the characteristics of human vision.

\section{SIFT Theory and Analysis}

Affine-invariant features have recently been studied in object recognition and image retrieval applications. These features are highly distinctive and can be matched with high probability under large image distortions. SIFT was proposed by Lowe [17/8 and has been proved to be robust to image rotation, scaling, translation, and to some extend illumination changes, and projective transforms. It has been applied to image forensics [1920, and digital watermarking [21]. The basic idea of SIFT is to extract features through a staged filtering that identifies stable feature points in the scale-space. In order to extract candidate locations for features, the scale-space is computed using Difference of Gaussian function, where an image is filtered by Gaussian function of different scales and then difference images are calculated. In this scale-space, all local maximum and minimum are retrieved by checking the eight closest neighbors at the same scale and nine neighbors at the scale above and the scale below [22. Finally the locations and descriptors of feature points are determined, using the scale and orientation changes. Some example images and their corresponding SIFT feature points are shown in Fig.2 (using the program provided by [23]). We can distinguish between edge areas and highly textured regions using the distribution 
of SIFT points, that is, the small number of points involved in edge areas can be regarded as noise that is supposed to be classified. This idea is the basis of this paper.

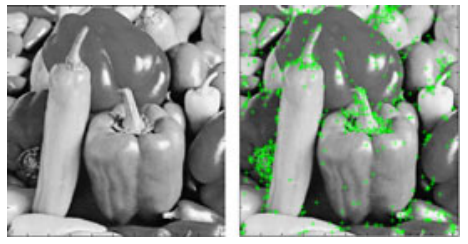

(a)

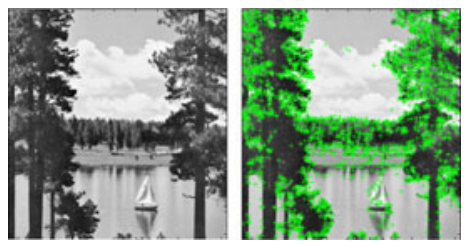

(c)

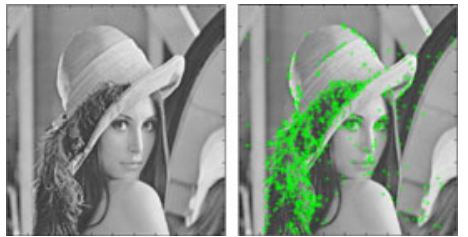

(b)

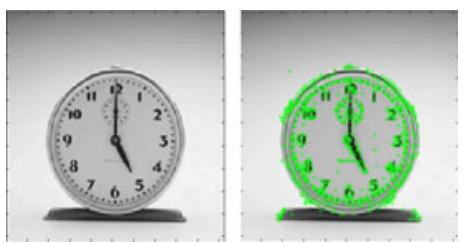

(d)

Fig. 2. Original images and the corresponding SIFT point locations

\section{Improved Method}

Our improved watermarking method has four steps,and is illustrated as Fig. 3 . Step1. SIFT extractor

Calculate feature points using the SIFT algorithm and obtain a binary siftmap for the arrangement of SIFT key points. We denote the pixel of cover image as $I(i, j), i=1,2, \ldots, M, j=1,2, \ldots, N$. The location set of feature points that are calculated with SIFT algorithm is denoted as $S$. The binary siftmap is denoted as $\mathbf{I}^{\prime}$, which is obtained by following formulation:

$$
I^{\prime}(i, j)= \begin{cases}1 & (i, j) \in S \\ 0 & \text { otherwise }\end{cases}
$$

Obviously, binary siftmap $\mathbf{I}^{\prime}$ is size of $M \times N$, the same with original cover image $\mathbf{I}$.

\section{Step2. Density-based clustering}

Cluster analysis is a primary method for database mining. The biggest advantage of density-based clustering is that regions indicated clusters may have an 


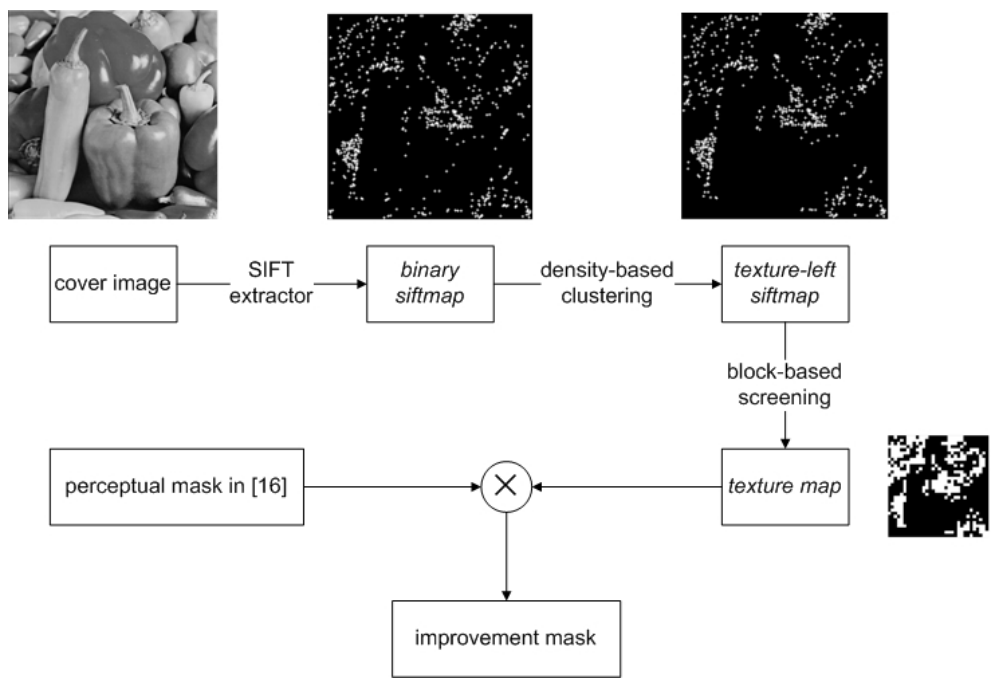

Fig. 3. Flow chart of proposed improvement approach and example result of every step

arbitrary shape and that the points inside a region may be arbitrarily distributed. In density-based clustering, clusters are regarded as regions in the data space in which the objects are dense, and which are separated by regions of low object density (noise). So density-based clustering is very suitable for classification of points on binary siftmap in the case of proposed improvement approach.

The first density-based clustering algorithm is proposed by Martin et al. in [24, which is called Density-Based Spatial Clustering of Applications with Noise (DBSCAN). It has two important parameters, search radius Eps and density search parameter MinPts. The details of the algorithm will not be described here and we only give the parameter settings. From adequate experiments we can find, the number of clusters is empirically supposed to be at the range from 6 to 30 in order to denoise to the greatest degree in this step. Moreover, clustering results are just sensitive to Eps, and MinPts can be constant. Therefore, the parameter Eps should be set carefully to make sure of getting a suitable number of clusters. We do experiments for an image database more than 30 and get an empirical range of the optimal search radius, that is, Eps $\in[10,16,20,32,40]$. The parameter MinPts is set to 4 .

A certain number of clusters is obtained after implement of DBSCAN. We denote these clusters as $C_{1}, C_{2}, \ldots, C_{k}$ and the number of points in them as $N_{u m}$, Num $_{2}, \ldots, N u m_{k}$. Then we sort the set of $N u m_{i}$ in accordance with descending order, which can be expressed as: 


$$
\operatorname{sort}\left(\left\{N u m_{1}, N_{u}, \ldots, N u m_{k}\right\}\right)=\left\{N u m_{1}^{*}, N u m_{2}^{*}, \ldots, N u m_{k}^{*}\right\}
$$

Thus permutation can be made to set of $C_{i}$ correspondingly and we can get $C_{1}^{*}, C_{2}^{*}, \ldots, C_{k}^{*}$. After all this, we can get texture-left siftmap, which is denoted as $\mathbf{I}^{*}$, through keeping the first $t$ of $C_{i}^{*}$ and discarding the points involved in other clusters on binary siftmap $\mathbf{I}^{\prime}$, which $t$ satisfies the following constrain:

$$
\sum_{i=1}^{t} N u m_{i}^{*} \geq p \sum_{i=1}^{k} N u m_{i}
$$

here $t$ is a natural number and $1<t<k, p$ is an given percent.

Step3. Block-based screening

As shown in Fig.4, the texture-left siftmap is a point-scattering mask so that it cannot be directly used as a perceptual mask in watermark embedding. We design a simple method to get a final texure map, which is called 'block-based density screening'. First of all, texture-left siftmap $\mathbf{I}^{*}$ (size of $M \times N$ ) is divided into non-overlapping small blocks. If the size of each block is defined as $m \times n$, the division process can be denoted as this expression:

$$
\mathbf{I}^{*}=\left[\begin{array}{cccc}
\mathbf{B}_{1,1} & \mathbf{B}_{1,2} & \ldots & \mathbf{B}_{1, \frac{N}{n}} \\
\mathbf{B}_{2,1} & \mathbf{B}_{2,2} & \ldots & \mathbf{B}_{2, \frac{N}{n}} \\
\vdots & \vdots & \ddots & \\
\mathbf{B}_{\frac{M}{m}, 1} & \mathbf{B}_{\frac{M}{m}, 2} & \ldots & \mathbf{B}_{\frac{M}{m}, \frac{N}{n}}
\end{array}\right]
$$

Then calculate the number of points in every corresponding block, which is expressed as:

$$
\left[\begin{array}{cccc}
\text { Num }_{1,1} & \text { Num }_{1,2} & \ldots & \text { Num }_{1, \frac{N}{n}} \\
\text { Num }_{2,1} & \text { Num }_{2,2} & \ldots & \text { Num }_{2, \frac{N}{n}} \\
\vdots & \vdots & \ddots & \\
\text { Num }_{\frac{M}{m}, 1} & \text { Num }_{\frac{M}{m}, 2} & \ldots & \text { Num }_{\frac{M}{m}, \frac{N}{n}}
\end{array}\right]
$$

The texture map, which is denoted as $\boldsymbol{T} \boldsymbol{M}$, can be obtained using following formulation:

$$
T M(i, j)= \begin{cases}1 & \text { Num }_{i, j}>\text { Threshold } \\ 0 & \text { Num }_{i, j} \leq \text { Threshold }\end{cases}
$$

Step4. Improved mask

For our research, the prototype of perceptual mask in [16] is improved using the texture map. Bilinear interpolation and binarization are sequentially applied to texture map to stretch and re-binarize it. Thus, a big texture map can be obtained with the same size of the prototype. In order to avoid the watermark information to be lost when the value of texture map $\boldsymbol{T M}$ equals to 0 , the final texture mask is processed by following rule: the values of pixels which equal to 
0 are set to a given small constant $t_{\text {min }}$ at the range of $(0,0.4]$; and the values of pixels which equal to 1 are unchanged. Finally, the perceptual mask (Equ.5) is modified as follows,

$$
\tilde{\Lambda}=\alpha \cdot(1-N V F) * \boldsymbol{T} \boldsymbol{M}+\beta \cdot N V F * \boldsymbol{T} \boldsymbol{M}
$$

here the operator $*$ does not represent matrix multiplication but array multiplication.

\section{Experiment Results}

\subsection{Improved Mask}

Experiments are performed on a set of images for our improvement approach. The parameter $p$ in the second step of our method is set to $90 \%$, Threshold in the third step is 1 , and $t_{\text {min }}$ in final step is 0.4 . Besides, $\alpha=250$ and $\beta=3$ is the same with Fig.1(c). For images in Fig.2, the comparison of perceptual masks is shown in Fig.4.

The advantage of our improved method is directly and clearly illustrated in Fig.4. Our method significantly decreases the JND values in all (or most) edge areas, and makes them have a considerable difference with JND values in highly textured regions. Before implement of proposed method, values of perceptual mask just have two levels, but the entire mask is to be divided into three levels after: the lowest in very flat region, the relatively lower in edge region, and the highest in highly-textured region. This advantage in the test image lake is most evident, particularly in parts of trunks and the small boat. Obviously, our perceptual masks are better for the characteristic of human vision than before.

\subsection{Application in Watermark Embedding}

We test the improved mask in the direct embedding method with the following equation,

$$
y=x+\tilde{\Lambda} \cdot \omega
$$

where $y$ denotes watermarked image, $x$ denotes cover image and $w$ denotes watermark. We use a grey-scale image (shown in Fig.5(a)) as watermark and embed it to a group of common test images. The embedding strength parameters $\alpha$ are set to 20 , and still $\beta=3$. The examples of cover image and watermarked image are shown in Fig.5(b) and (c).

The peak signal-to-noise ratio (PSNR) is chosen to measure the visibility of watermark and the quality of cover image. For an 8-bit grey-scale image, the equation of PSNR is as follows:

$$
P S N R=10 \log 10 \frac{255^{2}}{\frac{1}{M \times N} \sum_{i=1}^{M} \sum_{j=1}^{N}(y(i, j)-x(i, j))^{2}}
$$



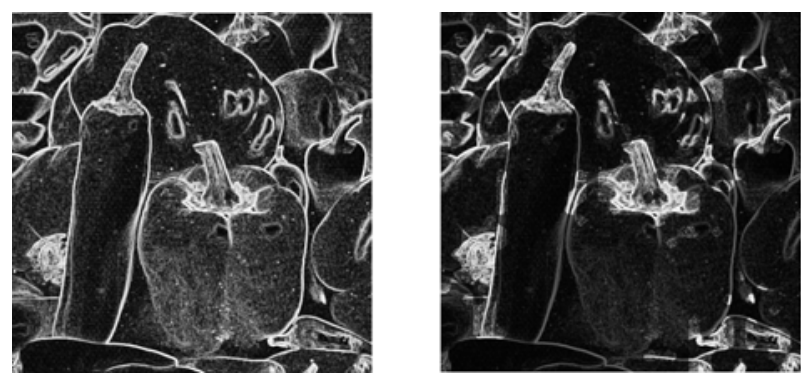

(a)
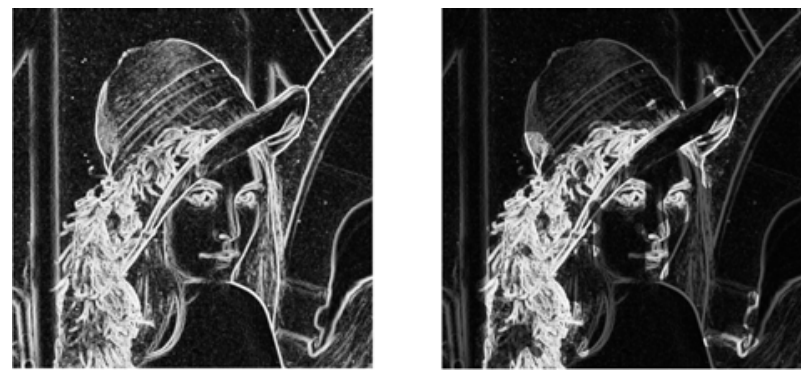

(b)
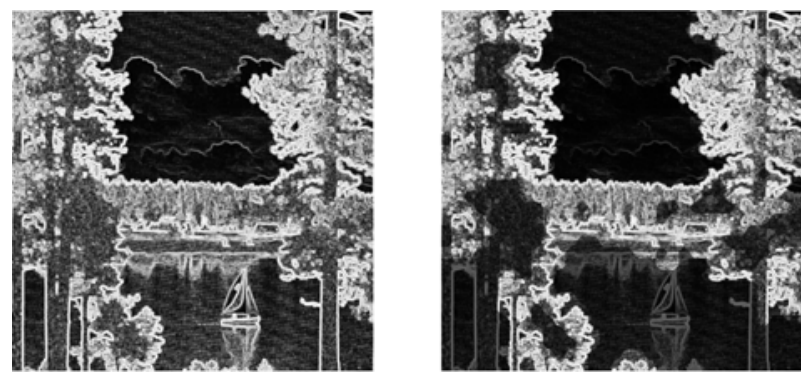

(c)

Fig. 4. Comparison of perceptual masks before and after implement of proposed improved method 


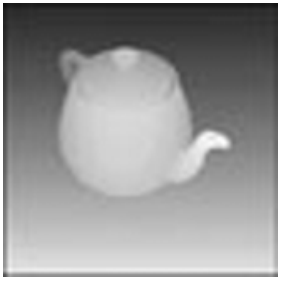

(a) watermark

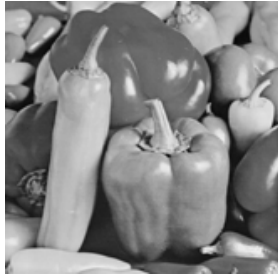

(b) cover image

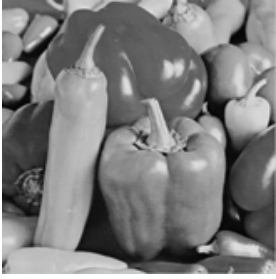

(c) watermarked image

Fig. 5. Watermark embedding

where $y(i, j)$ and $x(i, j)$ are the pixel value in watermarked image and original image respectively. $M \times N$ presents the image size. After watermark embedding, the values of PSNR calculated and parameters Eps for every image are listed in Table 1. More test results have been shown in Fig.6 for our image database. From these results we can find, our improved method significantly and generally increases the value of PSNR and enhances the invisibility of embedded watermark. Moreover, the more edge information cover image contains, the more of the PSNR value increases.

Table 1. Comparison of PSNR for mask effect in different methods

\begin{tabular}{|c|c|c|c|c|}
\hline cover image & Eps & PSNR(in [16]) & PSNR(here) & PSNR(no mask) \\
\hline peppers & 32 & $32.7540 \mathrm{db}$ & $35.5923 \mathrm{db}$ & $25.2939 \mathrm{db}$ \\
\hline lena & 24 & $31.5628 \mathrm{db}$ & $33.5113 \mathrm{db}$ & $25.7776 \mathrm{db}$ \\
\hline lake & 10 & $31.2367 \mathrm{db}$ & $32.6696 \mathrm{db}$ & $25.7413 \mathrm{db}$ \\
\hline clock & 10 & $33.5889 \mathrm{db}$ & $35.0734 \mathrm{db}$ & $26.3042 \mathrm{db}$ \\
\hline cameraman & 10 & $32.6604 \mathrm{db}$ & $33.5734 \mathrm{db}$ & $26.3042 \mathrm{db}$ \\
\hline baboon & 32 & $28.4366 \mathrm{db}$ & $29.1577 \mathrm{db}$ & $24.7816 \mathrm{db}$ \\
\hline
\end{tabular}

We also do experiments about watermarking attacks and calculate correlation values between original watermark and extracted watermark. The results against noise-adding (Salt \& pepper, Gaussian, Speckle) are illustrated in Fig.7. 


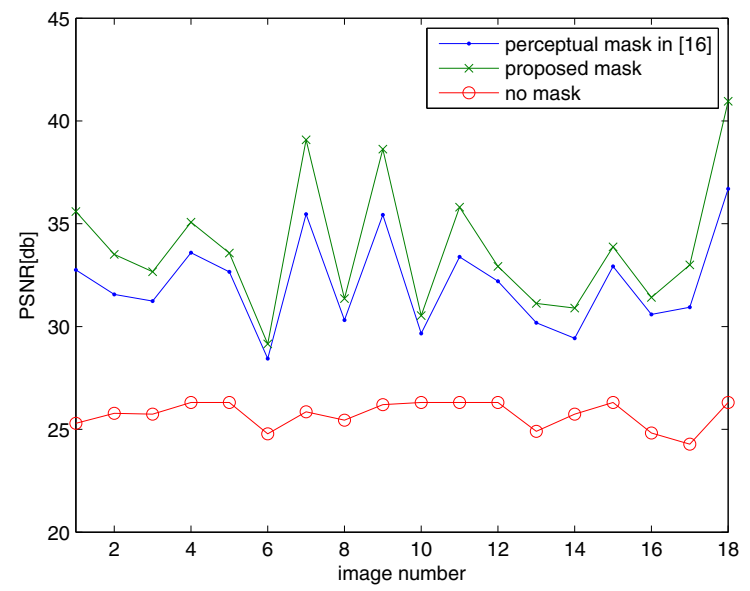

Fig. 6. Comparison of PSNR for image database

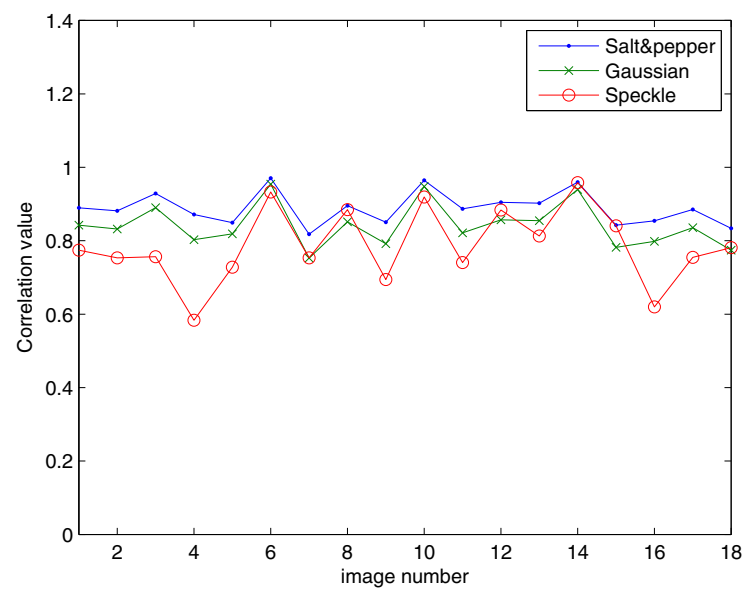

Fig. 7. Detection values of watermarked image after noise-adding attack

\section{Conclusion}

In this article, we describe an improved method for the perceptual mask using the arrangement of scale-invariant feature key points. It could decrease the JND values in edge areas of those traditional masks so that they give obvious difference with values in highly textured areas. Therefore, the improved mask is more suitable for human visual characteristics. But one of the shortcomings of our method is the imprecision of segment between the highly textured region and the edge areas. This is one of the directions of our future work. 


\section{References}

1. van Schyndel, R.G., Tirkel, A.Z., Osborne, C.F.: A digital watermark. In: Proceedings of 1st International Conference on Image Processing (ICIP 1994), vol. 2, pp. 86-90 (1994)

2. Cox, I.J., Miller, M.L.: A review of watermarking and the importance of perceptual modelling. In: Proc. SPIE Conf. on Human Vision and Electronic Imaging II, vol. 3016, pp. 92-99 (1997)

3. Nill, N.B.: A Visual Model Weighted Cosine Transform for Image Compression and Quality Assessment. IEEE Transactions on Communications 33(6), 551-557 (1985)

4. Jayant, N., Johnston, J., Safranek, B.: Signal compression based on models of human perception. Proceedings of the IEEE 81(10), 1385-1422 (1993)

5. Eckert, M.P., Bradley, A.P.: Perceptual quality metrics applied to still image compression. Signal Processing 70(3), 177-200 (1998)

6. Kankanhalli, M.S., Rajmohan, Ramakrishnan, K.R.: Content based watermarking of images. In: International Multimedia Conference in: Proceedings of the Sixth ACM International Conference on Multimedia, Bristol, United Kingdom, pp. 6170 (1998)

7. Delaigle, J.F., De Vleeschouwer, C., Macq, B.: Psychovisual approach to digital picture watermarking. Journal of Electronic Imaging 7(3), 628-640 (1998)

8. Delaigle, J.F., De Vleeschouwer, C., Macq, B.: Watermarking algorithm based on a human visual model. Signal Processing 66(3), 319-335 (1998)

9. Autrusseau, F., Le Callet, P.: A robust image watermarking technique based on quantization noise visibility thresholds. Signal Processing 87(6), 1363-1383 (2007)

10. Andrew, B.: Watson. DCT quantization matrices visually optimized for individual images. In: Proceedings of SPIE: Human vision, Visual Processing and Digital Display IV, vol. 1913, pp. 202-216

11. Lewis, A.S., Knowles, G.: Image compression using the 2-D wavelet transform. IEEE Trans. Image Processing 1, 244-250 (1992)

12. Barni, M., Bartolini, F., Piva, A.: Improved wavelet-based watermarking through pixel-wise masking. IEEE Transaction on Image Processing 10(5), 783-791 (2001)

13. Podilchuk, C.I., Zeng, W.: Image-adaptive watermarking using visual models. IEEE Journal on Selected Areas in Communications 16(4), 525-539 (1998)

14. Watson, A.B., Yang, G.Y., Solomon, J.A., Villasenor, J.D.: Visibility of wavelet quantization noise. IEEE Transactions on Image Processing 6(8), 1164-1175 (1997)

15. Qi, H., Zheng, D., Zhao, J.: Human visual system based adaptive digital image watermarking. Signal Processing 88(1), 174-188 (2008)

16. Voloshynovskiy, S., Herrigel, A., Baumgaertner, N., Pun, T.: A stochastic approach to content adaptive digital image watermarking. In: Pfitzmann, A. (ed.) IH 1999. LNCS, vol. 1768, pp. 212-236. Springer, Heidelberg (2000)

17. David, G.: Lowe. Object Recognition from Local Scale-Invariant Features. In: International Conference on Computer Vision, Corfu, Greece, pp. 1150-1157 (September 1999)

18. Lowe, D.G.: Distinctive Image Features from Scale-Invariant Keypoints. International Journal of Computer Vision 60(2), 91-110 (2004)

19. Amerini, I., Ballan, L., Caldelli, R., Del Bimbo, A., Serra, G.: A SIFT-based forensic method for copy-move attack detection and transformation recovery. IEEE Transactions on Information Forensics and Security (in press, 2011) 
20. Pan, X., Lyu, S.: Region Duplication Detection Using Image Feature Matching. IEEE Transactions on Information Forensics and Security 5(4), 857-867 (2010)

21. Nguyen, P.-B., Beghdadi, A., Luong, M.: Robust Watermarking in DoG Scale Space Using a Multi-scale JND Model. In: Muneesawang, P., Wu, F., Kumazawa, I., Roeksabutr, A., Liao, M., Tang, X. (eds.) PCM 2009. LNCS, vol. 5879, pp. 561573. Springer, Heidelberg (2009)

22. Lee, H.-Y., Lee, C.-h., Lee, H.-K., Nam, J.: Feature-Based Image Watermarking Method Using Scale-Invariant Keypoints. In: Ho, Y.-S., Kim, H.-J. (eds.) PCM 2005. LNCS, vol. 3768, pp. 312-324. Springer, Heidelberg (2005)

23. http://www.cs.ubc.ca/ lowe/keypoints/

24. Ester, M., Kriegel, H.-P., Sander, J., Xu, X.: A Density-Based Algorithm for Discovering Clusters in Large Spatial Databases with Noise. In: Proceedings of 2nd International Conference on Knowledge Discovery and Data Mining (KDD 1996), pp. 226-231 (1996) 